\title{
A Rare Case of Collision Tumor: Coexistence of Adrenocortical Adenoma and Pheochromocytoma in the Same Adrenal Gland
}

\author{
Samir Abdullazade ${ }^{a}$, Ilyas Sahinn, Gaye Guler Tezela, b
}

\begin{abstract}
Simultaneous occurrence of both adrenocortical adenoma and pheochromocytoma in the same adrenal gland is extremely rare in the literature. We report a 38-year-old female who presented with palpitation, chest pain and tremor with a right adrenal mass consistent with adrenocortical adenoma on computed tomography. Both metanephrine and normetanephrine levels were high in the urine. The biochemical tests strongly suggested the diagnosis of pheochromocytoma. Pathological examination of the adrenalectomy specimen revealed a collision tumor composed of an adrenocortical adenoma and a pheochromocytoma. Although rare, the present case is noteworthy in highlighting the importance of conducting biochemical tests to rule out pheochromocytoma for any adrenal mass prior to surgery.
\end{abstract}

Keywords: Adrenocortical adenoma; Pheochromocytoma; Adrenal; Collision tumor

\section{Introduction}

The adrenal gland is composed of two main parts with different embryologic origins. The inner core, referred to as the adrenal medulla, is derived from neural crest cells. The outer adrenal cortex is derived from the mesothelium. Neoplasms

Manuscript accepted for publication November 24, 2011

\footnotetext{
${ }^{a}$ Department of Pathology, Hacettepe University Hospital, 06100, Ankara, Turkey

${ }^{\mathrm{b}}$ Corresponding author: Gaye Guler Tezel, Department of Pathology, Hacettepe University Hospital, Sihhiye, Ankara, 06100, Turkey.

Email: gayejp@yahoo.com
}

may arise from both compartments and present with different clinical symptoms. Pheochromocytoma is a rare catecholamine secreting tumor derived from the chromaffin cells in the adrenal medulla, whereas adrenocortical adenoma is a benign epithelial tumor derived from adrenal cortical cells. Coexistence of adrenocortical adenoma and pheochromocytoma in the same gland is extremely rare. Only few cases have been reported in the English literature [1-9]. Herein, we report another case of adrenal collision tumor comprised of an adrenocortical adenoma and a pheochromocytoma in the same adrenal gland and emphasize the importance of conducting biochemical tests for pheochromocytoma before performing surgery to avoid intraoperative and postoperative complications of adrenal masses.

\section{Case Report}

A 38-year-old female patient was referred to the outpatient clinics of Hacettepe University Community Hospital with the complaint of palpitation and chest pain, as well as tremor present for the past couple of years. Six years ago she was diagnosed with nodular goiter and hypothyroidism. An abdominal computed tomography scan revealed a single bilobular mass in the right adrenal gland with prominent fatty components in the posterior part and intense opacification in the anterior part consistent with adrenocortical adenoma, whereas the left adrenal gland was normal. Elevated metanephrine and normetanephrine levels were detected in spot urine test. Right adrenalectomy was performed laparoscopically with the diagnosis of pheochromocytoma which was made upon biochemical, clinical and radiographic evaluation.

\section{Pathological Findings}

Right adrenalectomy specimen composed of three fragments having a total weight of 36 grams was examined (Fig. 1A). Macroscopically, the cut surface showed a brown nodule measuring $3 \mathrm{~cm}$ in diameter, a hemorrhagic nodule measuring $1 \mathrm{~cm}$ in diameter and a yellowish-orange nodule measuring $2.5 \mathrm{~cm}$ in diameter (Fig. 1B). Microscopically, the first two nodules consisted of neoplastic cells growing in

doi: $10.4021 / \mathrm{jmc} 451 \mathrm{w}$ 

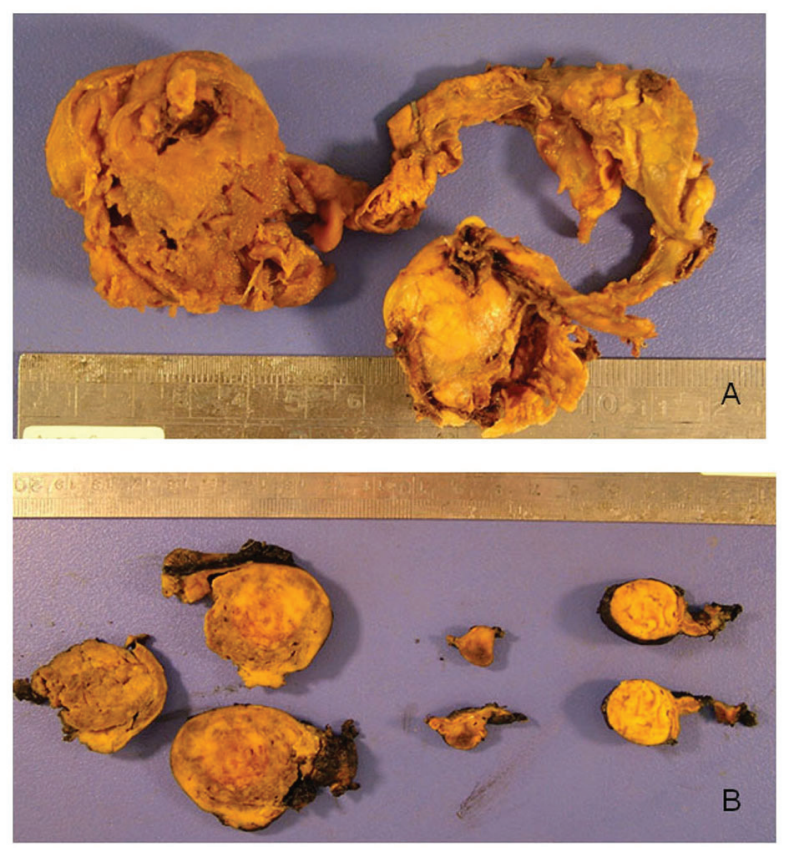

Figure 1. A: Macroscopic appearance of the "adrenal mass" specimen composed of three pieces with a total weight of 36 grams. B: The cut surfaces of the nodules.

a trabecular pattern with abundant eosinophilic cytoplasm and enlarged nuclei (Fig. 2A). These two nodules had fibrotic capsules demarcating them from the adrenal gland. Immunohistochemically, the neoplastic cells in both nodules diffusely stained with chromogranin (Fig. 2B) and sustantecular cells were positive for S-100 (Fig. 2C) whereas neo- plastic cells were negative for inhibin (Fig. 2D), calretinin and melan-A. Overall, these findings confirmed that the nodules had characteristic features of pheochromocytoma. Sections taken from the last nodule showed neoplastic cells with lipidized or eosinophilic cytoplasm and uniform nuclei (Fig. 3A). The nodule had a thin capsule separating it from
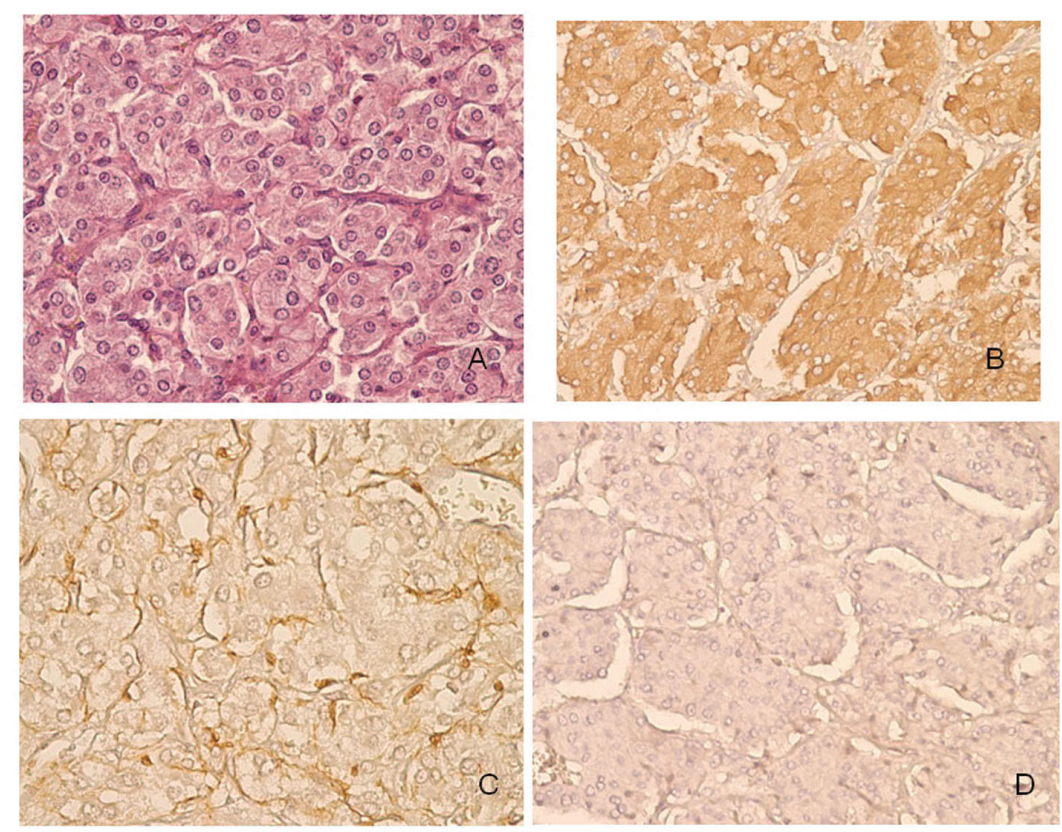

Figure 2. Histopathological features of pheochromocytoma of the adrenal gland in our patient. A: The trabecular architecture. B: The neoplastic cells diffusely stained with chromogranin. C: Immunoreactivity to S-100 in sustentacular cells. D: The neoplastic cells were negative for inhibin. 

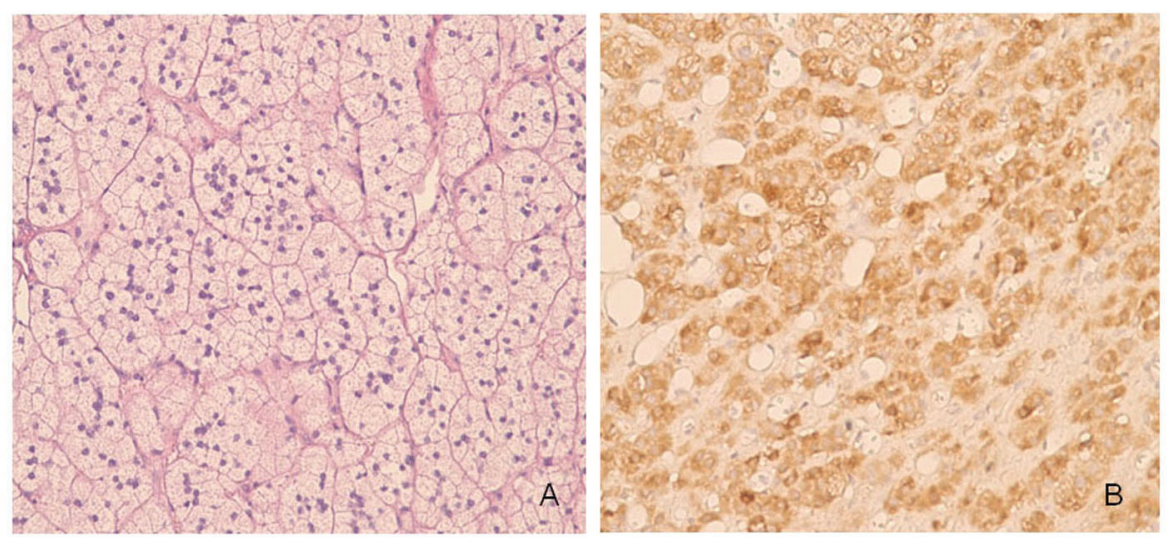

Figure 3. Histopathological features of adrenocortical adenoma of the adrenal gland in our patient. A: The neoplastic cells with intracytoplasmic lipid droplets or eosinophilic cytoplasm. B: The neoplastic cells were diffusely positive for inhibin.

the gland. The neoplastic cells were diffusely positive for inhibin (Fig. 3B), focally positive for calretinin and melan-A, whereas they were negative for chromogranin in immunohistochemical study. This nodule was diagnosed as an adrenocortical adenoma. The histologic findings of the nodule were also consistent with adrenocortical adenoma according to the criteria of Weiss. There was no evidence of necrosis, mitosis, capsular or vascular invasion in both neoplasms and both had a low Ki-67 proliferation index.

\section{Discussion}

There have been a number of reports in the English literature illustrating the coexistince of adrenocortical adenoma and pheochromocytoma in the same adrenal gland (Table

Table 1. Review of the English Literature for Adrenocortical Adenoma and Pheochromocytoma in the Same Adrenal Gland

\begin{tabular}{|c|c|c|c|c|c|c|}
\hline Case & Age & Gender & Year* & Clinical presentation & $\begin{array}{c}\text { Urine } \\
\text { catecholamine }\end{array}$ & Reference \\
\hline 1 & 55 & M & 1952 & Headache, hypertension & $\mathrm{N} / \mathrm{R}$ & 1 \\
\hline 2 & 56 & $\mathrm{~F}$ & 1977 & Hypertension & Elevated & 2 \\
\hline 3 & 46 & M & 1985 & Hypertension & Elevated & 3 \\
\hline 4 & 60 & $\mathrm{~F}$ & 1986 & Headache, hypertension, palpitation & Elevated & 4 \\
\hline 5 & 53 & M & 1988 & Fatigue, weight loss & Elevated & 5 \\
\hline 6 & 59 & $\mathrm{~F}$ & 2002 & Silent & Normal & 6 \\
\hline 7 & 43 & M & 2006 & Headache, hypertension, palpitation & Elevated & 7 \\
\hline 8 & 56 & M & 2006 & Hypertension, chest pain & Elevated & 7 \\
\hline 9 & 51 & $\mathrm{~F}$ & 2007 & Hypertension & Elevated & 8 \\
\hline 10 & 40 & $\mathrm{~F}$ & 2009 & Hypertension & $\mathrm{N} / \mathrm{R}$ & 9 \\
\hline 11 & 38 & $\mathrm{~F}$ & 2011 & Palpitation, chest pain & Elevated & Current \\
\hline
\end{tabular}

*The publication year of the reference; M: Male; F: Female; N/R: Not reported. 
1). To the best of our knowledge, the current case presents the eleventh of such cases. A corticomedullary mixed tumor, which has been defined as a single tumor mass originating from both adrenocortical and adrenal medullary cells, is also extremely rare [10].

A collision tumor is a rare condition in which two independent neoplasms without a substantial histologic admixture at the interface coexist adjacent to each other [11]. Based on this principle, the present case represents an extremely rare example of an unusual combination of adrenocortical adenoma and pheochromocytoma, collision type. The larger tissue specimen demonstrated neuroendocrine morphology, whereas the smaller tissue specimen showed typical characteristics of adrenocortical adenoma of the adrenal gland.

The most commonly described collision tumor in the adrenal gland is adrenal adenoma with myelolipoma. The other collision tumors reported in the adrenal glands include cases such as metastasis into an adenoma or myelolipoma and pheochromocytoma with adenoma or myelolipoma. Although collision tumors are rare, they have been reported in many other sites, including the meninges, the chest, the bow$\mathrm{el}$, the hepatobiliary system, the lymph nodes and the genitourinary tract [11]. Several hypotheses have been suggested to explain the mechanism of the collision tumors [12]. The occurrence of two primary tumors in continuity with each other incidentally is the simplest explanation. Alternatively, two different tumors may occur in contiguity because of similar alterations in the region induced by the same carcinogenic stimuli. Another possibility is that the changes created by the first tumor in the microenvironment may lead to the development of the second tumor. Since the adrenal cortex and medulla are different in their embryological origin, structure and function, it is hard to speculate on the pathogenesis of two different tumors. The release of corticotropin from pheochromocytoma is one of the major mechanisms in the development of adrenal hyperplasia and/or adenoma by stimulating the adrenal cortex. Another mechanism considered to take part in the development of adrenal hyperplasia and/or adenoma, is that the secretion of catecholamines by pheochromocytoma stimulate the anterior pituitary gland and in turn the release of corticotrophin [13]. However, it still remains unclear why most patients with pheochromocytoma do not have an accompanying adrenocortical adenoma in the adrenal glands.

Although pheochromocytomas are rare, occurring in $0.01-0.1 \%$ of hypertensive patients, it should be noted that they may have life-threatening consequences. The tumors occur most frequently in the 4 th and 5 th decades. The classical symptoms of pheochromocytoma include headache, palpitation, and diaphoresis in association with severe hypertension. However, one should keep in mind that some patients are totally asymptomatic while others have intermittent symptoms. The hallmark clinical manifestation of pheochromocytoma is hypertension seen in only about half of the patients, paroxysmal in about one third and absent in about one fifth [14]. The diagnosis of pheochromocytoma is usually established with biochemical studies, i.e., measurements of catecholamines and/or its metabolites in urine and/ or plasma, followed by clinic and radiographic evaluation. In the present case, although the presence of single bilobular mass at computed tomography (CT) suggested a diagnosis of an adrenocortical adenoma, biochemical tests performed prior to surgery strongly suggested a diagnosis of pheochromocytoma with elevated metanephrine and normetanephrine levels in the urine. Histopathological evaluation of the adrenalectomy material confirmed the presence of both adrenocortical adenoma and pheochromocytoma in the same gland.

\section{Conclusion}

In conclusion, even if the radiographic evaluation of an adrenal mass suggests adrenocortical adenoma, all clinicians need to be aware of the probability of pheochromocytoma to avoid intraoperative hypertensive crisis and/or postoperative adrenal insufficiency. Clinical suspicion is the single most important factor in the identification of pheochromocytoma. Thus, we strongly recommend conducting biochemical tests to rule out pheochromocytoma for every adrenal mass before surgery [15].

\section{References}

1. Cope O, Labbe JP, Raker JW, Bland EF. Pheochromocytoma and adrenal cortical adenoma; report of a case with both tumors and discussion of their relation. J Clin Endocrinol Metab. 1952;12(7):875-880.

2. Wilkins GE, Schmidt N, Lee-Son L. Coexistence of pheochromocytoma, adrenal adenoma and hypokalemia. Can Med Assoc J. 1977;116(4):360-362.

3. Wajiki M, Ogawa A, Fukui J, Komiya I, Yamada T, Maruyama Y. Coexistence of aldosteronoma and pheochromocytoma in an adrenal gland. J Surg Oncol. 1985;28(1):75-78.

4. Inoue J, Oishi S, Naomi S, Umeda T, Sato T. Pheochromocytoma associated with adrenocortical adenoma: case report and literature review. Endocrinol Jpn. 1986;33(1):67-74.

5. Aiba M, Hirayama A, Ito Y, Fujimoto Y, Nakagami Y, Demura H, Shizume K. A compound adrenal medullary tumor (pheochromocytoma and ganglioneuroma) and a cortical adenoma in the ipsilateral adrenal gland. A case report with enzyme histochemical and immunohistochemical studies. Am J Surg Pathol. 1988;12(7):559566.

6. Sato H, Igarashi H, Kishimoto Y, Yamaguchi K, Saito T, Ishida $\mathrm{H}$, Okada $\mathrm{K}$, et al. Combined tumor consisting of non-functioning adrenocortical adenoma and pheochro- 
mocytoma in the same gland. Int J Urol. 2002;9(7):398401.

7. Cotesta D, Petramala L, Serra V, Giustini S, Divona L, Calvieri S, De Toma G, et al. Pheochromocytoma associated with adrenocortical tumor in the same gland. Two case reports and literature review. Minerva Endocrinol. 2006;31(2):183-189.

8. Hwang WR, Ma WY, Tso AL, Pan CC, Chang YH, Lin HD. Pheochromocytoma and adrenocortical adenoma in the same gland. J Chin Med Assoc. 2007;70(7):289-293.

9. Sakamoto N, Tojo K, Saito T, Fujimoto K, Isaka T, Tajima N, Ikeda K, et al. Coexistence of aldosterone-producing adrenocortical adenoma and pheochromocytoma in an ipsilateral adrenal gland. Endocr J. 2009;56(2):213219.

10. Alexandraki KI, Michail OP, Nonni A, Diamantis D, Giannopoulou I, Kaltsas GA, Tseleni-Balafouta S, et al. Corticomedullary mixed adrenal tumor: case report and literature review. Endocr J. 2009;56(6):817-824.

11. Schwartz LH, Macari M, Huvos AG, Panicek DM. Collision tumors of the adrenal gland: demonstration and characterization at MR imaging. Radiology. 1996;201(3):757-760.

12. Brandwein-Gensler M, Urken M, Wang B. Collision tumor of the thyroid: a case report of metastatic liposarcoma plus papillary thyroid carcinoma. Head Neck. 2004;26(7):637-641.

13. Wieneke JA, Thompson LD, Heffess CS. Corticomedullary mixed tumor of the adrenal gland. Ann Diagn Pathol. 2001;5(5):304-308.

14. Arnaldi G, Masini AM, Giacchetti G, Taccaliti A, Faloia E, Mantero F. Adrenal incidentaloma. Braz J Med Biol Res. 2000;33(10):1177-1189.

15. Myklejord DJ. Undiagnosed pheochromocytoma: the anesthesiologist nightmare. Clin Med Res. 2004;2(1):5962. 